\title{
0563 INTEGRIS INTEGRATION OF EUROPEAN INJURY STATISTICS: FIRST RESULTS
}

R Bauer*, G Furian, B Laursen, R A Lyons, E Van Beeck Correspondence: Austrian Road Safety Board, Schleiergasse 18 A-1100 Wien Austria 1100, Austria

\subsection{6/ip.2010.029215.563}

The implementation of prevention-oriented injury surveillance in the European Union (EU) is a major demand towards the Member States in the recent EC Council Recommendations on the prevention of injury and the promotion of safety. Needed in particular are valid and reliable indicators for the array of external causes of injury morbidity, like involved activities, products and mechanisms, and for the most severe consequences of injuries besides death long term and chronic disabilities. Whereas no single EU health statistics fulfils that demand to date, the integration of two existing data sources the prevention-oriented European Injury Database (IDB) and the routine Hospital Discharge Registers (HDR) is considered the most promising approach to that avail. The RP7 funded INTEGRIS project which was started in May 2008 - aims to provide the necessary research and technology input for the IDB-HDR integration through an evaluated demonstration project with about 20 participating hospitals in six EU Member States. By the time of the conference first results from the pilot study will be available. As part of a live demonstration online queries of the 2010 integrated injury database - sourced from the participating hospitals - will be performed during the presentation, showing projections of regional and of national injury figures and indicators for injury related disability from the HDR medical information. By 2011 INTEGRIS shall result in an implementation plan for a cost-efficient injury surveillance system for all EU-member states that is compatible with the European Statistical System. 